\title{
A Variational Formula for Nonzero-Sum Stochastic Differential Games of FBSDEs and Applications
}

\author{
Maoning Tang \\ Department of Mathematical Sciences, Huzhou University, Zhejiang 313000, China \\ Correspondence should be addressed to Maoning Tang; tangmaoning@hutc.zj.cn
}

Received 4 December 2013; Revised 20 March 2014; Accepted 20 March 2014; Published 28 April 2014

Academic Editor: Gerhard-Wilhelm Weber

Copyright (C) 2014 Maoning Tang. This is an open access article distributed under the Creative Commons Attribution License, which permits unrestricted use, distribution, and reproduction in any medium, provided the original work is properly cited.

\begin{abstract}
A nonzero-sum stochastic differential game problem is investigated for fully coupled forward-backward stochastic differential equations (FBSDEs in short) where the control domain is not necessarily convex. A variational formula for the cost functional in a given spike perturbation direction of control processes is derived by the Hamiltonian and associated adjoint systems. As an application, a global stochastic maximum principleof Pontryagin's type for open-loop Nash equilibrium points is established. Finally, an example of a linear quadratic nonzero-sum game problem is presented to illustrate that the theories may have interesting practical applications and the corresponding Nash equilibrium point is characterized by the optimality system. Here the optimality system is a fully coupled FBSDE with double dimensions (DFBSDEs in short) which consists of the state equation, the adjoint equation, and the optimality conditions.
\end{abstract}

\section{Introduction}

Bismut [1] first investigated linear backward stochastic differential equations (BSDEs in short) as the adjoint equation of the forward stochastic system. The existence and uniqueness of BSDEs with nonlinear generators under Lipschitz condition were first proved by Pardoux and Peng [2] in 1990. Since then, the theory of BSDEs has extensive applications in both mathematical finance and stochastic control.

Forward-backward stochastic differential equations (FBSDEs in short) consist of forward stochastic differential equations (SDEs in short) of Itô type and BSDEs of PardouxPeng. The main motivations of studying FBSDEs mainly come from stochastic control theories and practical applications of finance. In the stochastic optimal control problem, FBSDEs arise as the Hamilton system which is composed of the optimality conditions, the adjoint equation, and the state equation; see, for example, [3]. In mathematical finance, FBSDEs can be formulated as the price equations of financial assets in model uncertainty and risk minimizing strategy for economic management problems; see, for example, [4, 5]. It now becomes more clear that certain important problems in mathematical economics and mathematical finance, especially in the optimization problem, can be formulated to be forward-backward stochastic system.

It is well known that in an optimal control problem, there is single control and single criterion to be optimized. And the so-called differential game is to generalize single control and single criterion in the optimal control problem to two controls and two criteria, one for each player. Each player attempts to control the state of the system so as to achieve his goal. The optimal control problem for forwardbackward stochastic system is extensively studied; see, for example, [6-12] and references therein. But, to the best of our knowledge, very little work has been published to discuss the maximum principle of stochastic differential games for forward-backward stochastic systems. In 2012, Hui and Xiao [13] established the maximum principle of differential games for forward-backward stochastic systems under convex control domain by means of a convex variation method and a duality technique. And in 2012, Øksendal and Sulem [5] studied optimal control problems with jumps under model uncertainty and partial information. They rewrite such problems as stochastic differential games of FBSDEs and obtained the corresponding stochastic maximum principle 
and verification theorem under convex control domain. Different from the decoupled forward-backward stochastic system studied in $[5,13]$, Tang [14] studied differential games for fully coupled FBSDEs under convex control domain and established the local maximum principle and the verification theorem. In this paper, we will also consider twoperson nonzero differential games of fully coupled forwardbackward stochastic systems. Different from [5, 13, 14], the control domain discussed in this paper is not necessarily convex. The main contribution of our paper is to get directly a variation formula for the cost functional in a given spike perturbation direction of control processes in terms of the Hamiltonian and the associated adjoint system which is a linear FBSDEs, and neither the variational systems nor the corresponding Taylor type expansions of the state process and the cost functional will be considered. As an application, a global stochastic maximum principle for open-loop Nash equilibrium points is established. And as a result, a linear quadratic nonzero-sum game problem is studied to illustrate that the established theories and the corresponding Nash equilibrium point are characterized by the optimality system.

The paper is organized as follows. In Section 2, we formulate the problem and give various assumptions used throughout the paper. In Section 3, we obtain the representation for the difference of the performance functional in terms of the Hamilton and adjoint processes. In Section 4, we use the representation in Section 3 to derive a representation for the variation and "directional derivative" of the difference of the performance functional along with spike variation. Section 5 is devoted to deriving the global stochastic maximum principles by the "directional derivative" formula established in Section 4. A linear quadratic nonzero-sum game problem is studied in Section 6. In Section 7, we conclude this paper.

\section{Formulation of the Problem}

Let $\left(\Omega, \mathscr{F},\left\{\mathscr{F}_{t}\right\}_{t \geq 0}, P\right)$ be a complete probability space, on which a $d$-dimensional standard Brownian motion $B(\cdot)$ is defined with $\left\{\mathscr{F}_{t}\right\}_{t>0}$ being its natural filtration, augmented by all $P$-null sets in $\mathscr{F}$. Let $T>0$ be a fixed time horizon. Let $E$ be a Euclidean space. The inner product in $E$ is denoted by $\langle\cdot, \cdot\rangle$, and the norm in $E$ is denoted by $|\cdot|$. We further introduce some other spaces that will be used in the paper. Denote by $L^{2}\left(\Omega, \mathscr{F}_{T}, P ; E\right)$ the set of all $E$-valued $\mathscr{F}_{T^{-}}$ measurable random variable $\eta$ such that $\mathbb{E}|\eta|^{2}<\infty$. Denote by $M_{\mathscr{F}}^{2}(0, T ; E)$ the set of all $E$-valued $\mathscr{F}_{t}$-adapted stochastic processes $\{\varphi(t): t \in[0, T]\}$ which satisfy $\mathbb{E} \int_{0}^{T}|\varphi(t)|^{2} d t<$ $\infty$. Denote by $\mathcal{S}_{\mathscr{F}}^{2}(0, T ; E)$ set of all $E$-valued $\mathscr{F}_{t}$-adapted continuous stochastic processes $\{\varphi(t): t \in[0, T]\}$ which satisfy $\mathbb{E}_{\sup _{0 \leq t \leq T}}|\varphi(t)|^{2}<\infty$. Finally, we define $\mathbb{M}^{2}[0, T]=$ $S_{\mathscr{F}}^{2}\left(0, T ; \mathbb{R}^{n}\right) \times S_{\mathscr{F}}^{2}\left(0, T ; \mathbb{R}^{m}\right) \times M_{\mathscr{F}}^{2}\left(0, T ; \mathbb{R}^{m \times d}\right)$. Then $\mathbb{M}^{2}[0, T]$ is a Banach space with respect to the norm $\|\cdot\|_{\mathbb{M}^{2}}$ given by $\|\Theta(\cdot)\|_{\mathbb{M}^{2}}^{2}=\mathbb{E}_{\sup _{0 \leq t \leq T}}|x(t)|^{2}+\mathbb{E}_{\sup _{0 \leq t \leq T}}|y(t)|^{2}+\mathbb{E} \int_{0}^{T}|z(t)|^{2} d t$ for $\Theta(\cdot)=(x(\cdot), y(\cdot), z(\cdot)) \in \mathbb{M}^{2}[0, T]$.

In the following, we specify two-person nonzero-sum differential game problem of fully coupled forward-backward stochastic systems. More precisely, for $a \in \mathbb{R}^{n}$, we consider a fully coupled nonlinear FBSDE

$$
\begin{aligned}
x(t)= & a+\int_{0}^{t} b\left(s, x(s), y(s), z(s), u_{1}(s), u_{2}(s)\right) d s \\
& +\int_{0}^{t} \sigma(s, x(s), y(s), z(s)) d B(s), \\
y(t)= & h(x(T))+\int_{t}^{T} f\left(s, x(s), y(s), z(s), u_{1}(s), u_{2}(s)\right) d s \\
& -\int_{t}^{T} z(s) d B(s) .
\end{aligned}
$$

The processes $u_{1}(\cdot)$ and $u_{2}(\cdot)$ in the system (1) are the openloop control processes which present the controls of the two players.

For each one of the two players, there is a cost functional

$$
\begin{aligned}
& J_{i}\left(u_{1}(\cdot), u_{2}(\cdot)\right) \\
& =E\left[\int_{0}^{T} l_{i}\left(t, x(t), y(t), z(t), u_{1}(t), u_{2}(t)\right) d t\right. \\
& \left.\quad+\phi_{i}(x(T))+\gamma_{i}(y(0))\right], \quad i=1,2 .
\end{aligned}
$$

In the above, $b:[0, T] \times \mathbb{R}^{n} \times \mathbb{R}^{m} \times \mathbb{R}^{m \times d} \times U_{1} \times U_{2} \rightarrow \mathbb{R}^{n}$, $\sigma:[0, T] \times \mathbb{R}^{n} \times \mathbb{R}^{m} \times \mathbb{R}^{m \times d} \rightarrow \mathbb{R}^{n \times d}, f:[0, T] \times$ $\mathbb{R}^{n} \times \mathbb{R}^{m} \times \mathbb{R}^{m \times d} \times U_{1} \times U_{2} \rightarrow \mathbb{R}^{m}, h: \mathbb{R}^{n} \rightarrow \mathbb{R}^{m}$, and $l_{i}:[0, T] \times \mathbb{R}^{n} \times \mathbb{R}^{m} \times \mathbb{R}^{m \times d} \times U_{1} \times U_{2} \rightarrow R, \phi_{i}$ : $\mathbb{R}^{n} \rightarrow \mathbb{R}, \gamma_{i}: \mathbb{R}^{m} \rightarrow \mathbb{R}$ are given Borel measurable mapping $(i=1,2)$. Here $U_{1} \subset \mathbb{R}^{k_{1}}$ and $U_{2} \subset \mathbb{R}^{k_{2}}$ are nonempty Borel subsets. The admissible control process $\left(u_{1}(\cdot), u_{2}(\cdot)\right)$ is defined as a $\mathscr{F}_{t}$-adapted process with values in $U_{1} \times U_{2}$ such that $\sup _{0 \leq t \leq T} \mathbb{E}\left[\left|u_{1}(t)\right|^{2}+\left|u_{2}(t)\right|^{2}\right]<\infty$. The set of all admissible control processes is denoted by $\mathscr{A}_{1} \times \mathscr{A}_{2}$.

Before giving the basic assumptions on the coefficients throughout this paper, we first introduce some abbreviations. Let $G$ be a given $m \times n$ full-rank matrix. Denote $\theta=\left(\begin{array}{l}x \\ y \\ z\end{array}\right)$ and $A(t, \theta)=\left(\begin{array}{c}-G^{*} f \\ G b \\ G \sigma\end{array}\right)$, where $G^{*}$ is the transpose matrix of $G$. For all $\theta=(x, y, z)$ and $\bar{\theta}=(\bar{x}, \bar{y}, \bar{z})$, we denote $\widehat{x}=x-\bar{x}$, $\widehat{y}=y-\bar{y}, \widehat{z}=z-\bar{z}$.

Assumption 1. (i) The mappings $f, b, \sigma$, and $h$ are continuously differentiable with respect to $(x, y, z)$ and the corresponding derivatives are bounded. Moreover, $f, b$, and $\sigma$ are bounded by $\left(1+|x|+|y|+\left|u_{1}\right|+\left|u_{2}\right|\right)$, and $h$ is bounded by $(1+|x|)$.

(ii) The mappings $f, b, \sigma$, and $h$ satisfy the following Monotonicity conditions:

$$
\begin{aligned}
& \langle A(t, \theta, u)-A(t, \bar{\theta}, u), \theta-\bar{\theta}\rangle \\
& \leq-\beta_{1}|G \hat{x}|^{2}-\beta_{2}\left[\left|G^{*} \hat{y}\right|^{2}+\left|G^{*} \hat{z}\right|^{2}\right], \\
& \langle h(x)-h(\bar{x}), x-\bar{x}\rangle \geq \mu_{1}|G \hat{x}|^{2},
\end{aligned}
$$


or

$$
\begin{gathered}
\langle A(t, \theta, u)-A(t, \bar{\theta}, u), \theta-\bar{\theta}\rangle \\
\geq \beta_{1}|G \hat{x}|^{2}+\beta_{2}\left(\left|G^{*} \hat{y}\right|^{2}+\left|G^{*} \hat{z}\right|^{2}\right), \\
\langle h(x)-h(\bar{x}), x-\bar{x}\rangle \leq-\mu_{1}|G \hat{x}|^{2} .
\end{gathered}
$$

Here $\beta_{1}, \beta_{2}$, and $\mu_{1}$ are given nonnegative constants with $\beta_{1}+$ $\beta_{2}>0, \beta_{2}+\mu_{1}>0$. Moreover, we have $\beta_{1}>0, \mu_{1}>0$ (resp., $\left.\beta_{2}>0\right)$, when $m>n$ (resp. $\left.m<n\right)$.

(iii) The mappings $l_{i}, \phi_{i}$, and $\gamma_{i}$ are continuously differentiable with respect to $(x, y, z),(i=1,2)$. And $l_{i}$ is bounded by $\left(1+|x|^{2}+|y|^{2}+|z|^{2}+\left|u_{1}\right|^{2}+\left|u_{2}\right|^{2}\right)$. And the derivatives of $l_{i}$ with respect to $(x, y, z),(i=1,2)$ are bounded by $(1+|x|+$ $\left.|y|+|z|+\left|u_{1}\right|+\left|u_{2}\right|\right)$. And $\phi_{i}$ and $h_{i}$ are bounded by $\left(1+|x|^{2}\right)$ and $\left(1+|y|^{2}\right)$, respectively. And the derivatives of $\phi_{i}$ and $h_{i}$ with respect to $x$ and $y$ are bounded by $(1+|x|)$ and $(1+|y|)$, respectively $(i=1,2)$.

Under Assumption 1, we see that for any given admissible control $u(\cdot)=\left(u_{1}(\cdot), u_{2}(\cdot)\right) \in \mathscr{A}_{1} \times \mathscr{A}_{2}$, the system (1) admits a unique solution $\left(x^{u}(\cdot), y^{u}(\cdot), z^{u}(\cdot)\right) \in \mathbb{M}^{2}$ (see [15]). Then we call $\left(x^{u}(\cdot), y^{u}(\cdot), z^{u}(\cdot)\right)$, or $(x(\cdot), y(\cdot), z(\cdot))$ if its dependence on admissible control $u(\cdot)$ is clear from context, the state process corresponding to the control process $u(\cdot)=\left(u_{1}(\cdot), u_{2}(\cdot)\right)$ and $(u(\cdot) ; x(\cdot), y(\cdot), z(\cdot))$ the admissible pair.

Then we can pose the following two-person nonzero-sum stochastic differential game problem.

Problem 2. Find an open-loop admissible control $\left(\bar{u}_{1}(\cdot), \bar{u}_{2}(\cdot)\right) \in \mathscr{A}_{1} \times \mathscr{A}_{2}$ such that

$$
\begin{aligned}
& J_{1}\left(\bar{u}_{1}(\cdot), \bar{u}_{2}(\cdot)\right)=\inf _{u_{1}(\cdot) \in \mathscr{A}_{1}} J_{1}\left(u_{1}(\cdot), \bar{u}_{2}(\cdot)\right), \\
& J_{2}\left(\bar{u}_{1}(\cdot), \bar{u}_{2}(\cdot)\right)=\inf _{u_{2}(\cdot) \in \mathscr{A}_{2}} J_{2}\left(\bar{u}_{1}(\cdot), u_{2}(\cdot)\right) .
\end{aligned}
$$

Any $\left(\bar{u}_{1}(\cdot), \bar{u}_{2}(\cdot)\right) \in \mathscr{A}_{1} \times \mathscr{A}_{2}$ satisfying the above is called an open-loop Nash equilibrium point of Problem 2. Such an admissible control allows two players to play individual optimal control strategies simultaneously.

\section{Representation for Difference of the Cost Functional}

This section is devoted to establishing a representation for the difference of the cost functional according to Hamiltonian and the adjoint processes of Problem 2.

To simplify our notation, for any admissible control $\bar{u}(\cdot)=\left(\bar{u}_{1}(\cdot), \bar{u}_{2}(\cdot)\right)$, we write $\bar{\Theta}(t):=(\bar{x}(t), \bar{y}(t), \bar{z}(t))$ as the corresponding state process. We define the Hamiltonian functions $H_{i}:[0, T] \times \mathbb{R}^{n} \times \mathbb{R}^{m} \times \mathbb{R}^{m \times d} \times \mathcal{U}_{1} \times \mathcal{U}_{2} \times \mathbb{R}^{n} \times$ $\mathbb{R}^{n \times d} \times \mathbb{R}^{m} \rightarrow \mathbb{R}$ by

$$
\begin{aligned}
H_{i}\left(t, x, y, z, u_{1}, u_{2}, p, q, k\right)= & \left\langle k,-f\left(t, x, y, z, u_{1}, u_{2}\right)\right\rangle \\
& +\left\langle p, b\left(t, x, y, z, u_{1}, u_{2}\right)\right\rangle
\end{aligned}
$$

$$
\begin{aligned}
& +l_{i}\left(t, x, y, z, u_{1}, u_{2}\right) \\
& +\langle q, \sigma(t, x, y, z)\rangle, \quad i=1,2 .
\end{aligned}
$$

For any admissible pair $(\bar{u}(\cdot) ; \bar{\Theta}(\cdot))$, we define the corresponding adjoint process $\Lambda_{i}^{\bar{u}}(\cdot)=\left(k_{i}^{\bar{u}}(\cdot), p_{i}^{\bar{u}}(\cdot), q_{i}^{\bar{u}}(\cdot)\right)(i=1,2)$ as the solution to the following FBSDEs:

$$
\begin{gathered}
d k_{i}(t)=-\bar{H}_{i y}(t) d t-\bar{H}_{i z}(t) d B(t), \\
d p_{i}(t)=-\bar{H}_{i x}(t) d t+q_{i}(t) d B(t), \\
k_{i}(0)=-\gamma_{i y}(\bar{y}(0)), \\
p_{i}(T)=-h_{x}^{*}(\bar{x}(T)) k_{i}(T)+\phi_{i x}(\bar{x}(T)), \\
i=1,2,
\end{gathered}
$$

where we have used the short hand notation

$$
\bar{H}_{i y}(t)=H_{i y}\left(t, \bar{u}(t), \bar{\Theta}(t), \Lambda_{i}^{\bar{u}}(t)\right), \quad i=1.2 .
$$

And similarly we can define $\bar{H}_{i x}(t)$ and $\bar{H}_{i z}(t)$.

Under Assumptions 1, it is easy to see that the above adjoint equations have unique solution $\Lambda_{i}^{\bar{u}}(\cdot):=\left(k_{i}^{\bar{u}}(\cdot), p_{i}^{\bar{u}}(\cdot), q_{i}^{\bar{u}}(\cdot)\right) \in \mathbb{M}^{2}[0, T], i=1,2$.

Now let $\left(u^{\varepsilon}(\cdot) ; \Theta^{\varepsilon}(\cdot)\right)=\left(u_{1}^{\varepsilon}(\cdot), u_{2}^{\varepsilon}(\cdot) ; x^{\varepsilon}(\cdot), y^{\varepsilon}(\cdot), z^{\varepsilon}(\cdot)\right)$ be another admissible pair. In the following we will give the presentation for the difference $J_{i}\left(u^{\varepsilon}(\cdot)\right)-J_{i}(\bar{u}(\cdot))(i=1,2)$ in terms of the Hamiltonian $H_{i}(i=1,2)$ and adjoint process $\Lambda_{i}^{\bar{u}}(\cdot)(i=1,2)$ associated with the admissible control pair $\left(\bar{u}(\cdot) ; \Theta^{\bar{u}}(\cdot)\right)$, as well as other relevant expressions. We state our result as follows.

Theorem 3. Under Assumptions 1, one has the representation for the difference of the cost functional as follows: $\cdot\left(y^{\varepsilon}(0)-\right.$ $\left.\bar{y}(0))\left(y^{\varepsilon}(0)\right)\right]$

$$
\begin{aligned}
J_{i}\left(u_{1}^{\varepsilon}(\cdot), u_{2}^{\varepsilon}(\cdot)\right) & -J_{i}\left(u_{1}(\cdot), u_{2}(\cdot)\right) \\
=\mathbb{E} \int_{0}^{T}\left[H_{i}(\right. & \left.t, \bar{\Theta}(t), u^{\varepsilon}(t), \Lambda_{i}^{\bar{u}}(t)\right) \\
& -H_{i}\left(t, \bar{\Theta}(t), \bar{u}(t), \Lambda_{i}^{\bar{u}}(t)\right) \\
& +H_{i}\left(t, \Theta^{\varepsilon}(t), u^{\varepsilon}(t), \Lambda_{i}^{\bar{u}}(t)\right) \\
& -H_{i}\left(t, \bar{\Theta}(t), u^{\varepsilon}(t), \Lambda_{i}^{\bar{u}}(t)\right) \\
& -\left\langle\bar{H}_{i x}(t), x^{\varepsilon}(t)-\bar{x}(t)\right\rangle \\
& -\left\langle\bar{H}_{i y}(t), y^{\varepsilon}(t)-\bar{y}(t)\right\rangle \\
- & \left.\left\langle\bar{H}_{i z}(t), z^{\varepsilon}(t)-\bar{z}(t)\right\rangle\right] d t
\end{aligned}
$$




$$
\begin{gathered}
+\mathbb{E}\left[\gamma_{i}\left(y^{\varepsilon}(0)\right)-\gamma_{i}(\bar{y}(0))-\gamma_{i y}(\bar{y}(0))\right. \\
\left.\cdot\left(y^{\varepsilon}(0)-\bar{y}(0)\right)\left(y^{\varepsilon}(0)\right)\right] \\
+\mathbb{E}\left[\phi_{i}\left(x^{\epsilon}(T)\right)-\phi_{i}(\bar{x}(T))-\phi_{i x}(\bar{x}(T))\right. \\
\left.\cdot\left(x^{\varepsilon}(T)-\bar{x}(T)\right)\right] \\
-\mathbb{E}\left[\left\langle h\left(x^{\epsilon}(T)\right)-h(\bar{x}(T)), k_{i}^{\bar{u}}(T)\right\rangle\right. \\
\left.-\left\langle h_{x}(\bar{x}(T))\left(x^{\varepsilon}(T)-\bar{x}(T)\right), k_{i}^{\bar{u}}(T)\right\rangle\right] .
\end{gathered}
$$

Proof. First, from the definition of $H$ (see (7)), it is easy to check that

$$
\begin{aligned}
H_{i}\left(t, \Theta^{\varepsilon}(t), u^{\varepsilon}(t), \Lambda_{i}^{\bar{u}}(t)\right)-H_{i}\left(t, \bar{\Theta}(t), \bar{u}(t), \Lambda_{i}^{\bar{u}}(t)\right) \\
=H_{i}\left(t, \bar{\Theta}(t), u^{\varepsilon}(t), \Lambda_{i}^{\bar{u}}(\cdot)\right)-H_{i}\left(t, \bar{\Theta}(t), \bar{u}(t), \Lambda_{i}^{\bar{u}}(t)\right) \\
\quad+H_{i}\left(t, \Theta^{\varepsilon}(t), u^{\varepsilon}(t), \Lambda_{i}^{\bar{u}}(t)\right) \\
\quad-H_{i}\left(t, \bar{\Theta}(t), u^{\varepsilon}(t), \Lambda_{i}^{\bar{u}}(t)\right), \quad i=1,2 .
\end{aligned}
$$

From (1), we know that

$$
\begin{aligned}
x^{\varepsilon}(t)-\bar{x}(t)= & \int_{0}^{t}\left[b\left(s, \Theta^{\varepsilon}(s), u^{\varepsilon}(s)\right)\right. \\
& -b(s, \bar{\Theta}(s), \bar{u}(s))] d s \\
& +\int_{0}^{t}\left[\sigma\left(s, \Theta^{\varepsilon}(s), u^{\varepsilon}(s)\right)\right. \\
y^{\varepsilon}(t)-\bar{y}(t)= & \left.h\left(x^{\epsilon}(T)\right)-h(s, \bar{\Theta}(s), \bar{u}(s))\right] d W(s), \\
& +\int_{t}^{T}\left[f\left(s, \Theta^{\varepsilon}(s), u^{\varepsilon}(s)\right)\right. \\
& -\int_{t}^{T}\left[z^{\varepsilon}(s)-\bar{z}(s)\right] d W(s) .
\end{aligned}
$$

Furthermore, recalling (8) and applying Itô formula to $\left\langle k_{i}^{\bar{u}}(t), y^{\varepsilon}(t)-\bar{y}(t)\right\rangle+\left\langle p_{i}^{\bar{u}}(t), x^{\varepsilon}(t)-\bar{x}(t)\right\rangle$, we deduce that

$$
\begin{gathered}
\mathbb{E} \int_{0}^{T}\left\langle k_{i}^{\bar{u}}(t),-\left(f\left(t, \Theta^{\varepsilon}(t), u^{\varepsilon}(t)\right)\right.\right. \\
-f(t, \bar{\Theta}(t), \bar{u}(t)))\rangle d t \\
+E \int_{0}^{T}\left\langle p_{i}^{\bar{u}}(t), b\left(t, \Theta^{\varepsilon}(t), u^{\varepsilon}(t)\right)\right. \\
-b(t, \bar{\Theta}(t), \bar{u}(t))\rangle d t
\end{gathered}
$$

$$
\begin{aligned}
+E \int_{0}^{T} & \left\langle q_{i}^{\bar{u}}(t), \sigma\left(t, \Theta^{\varepsilon}(t), u^{\varepsilon}(t)\right)\right. \\
& -\sigma(t, \bar{\Theta}(t), \bar{u}(t))\rangle d t \\
=\mathbb{E} \int_{0}^{T}[ & \left\langle\bar{H}_{i x}(t), x^{\varepsilon}(t)-\bar{x}(t)\right\rangle \\
& +\left\langle\bar{H}_{i y}(t), y^{\varepsilon}(t)-\bar{y}(t)\right\rangle \\
& \left.+\left\langle\bar{H}_{i z}(t), z^{\varepsilon}(t)-\bar{z}(t)\right\rangle\right] d t
\end{aligned}
$$

$$
\begin{aligned}
+ & \mathbb{E}\left[\gamma_{i y}(\bar{y}(0)) \cdot\left(y^{\varepsilon}(0)-\bar{y}(0)\right)\right] \\
+ & \mathbb{E}\left[\phi_{i x}(\bar{x}(T)) \cdot\left(x^{\varepsilon}(T)-\bar{x}(T)\right)\right] \\
+ & \mathbb{E}\left[\left\langle h\left(x^{\varepsilon}(T)\right)-h(\bar{x}(T)), k_{i}^{\bar{u}}(T)\right\rangle\right. \\
& \left.-\left\langle h_{x}(\bar{x}(T))\left(x^{\varepsilon}(T)-\bar{x}(T)\right), k_{i}^{\bar{u}}(T)\right\rangle\right],
\end{aligned}
$$$$
i=1,2 \text {. }
$$

On the other hand, by the definition of the Hamilton function $H$ (see (7)), we deduce that

$$
\begin{aligned}
& J_{i}\left(u_{1}^{\varepsilon}(\cdot), u_{2}^{\varepsilon}(\cdot)\right)-J_{i}\left(u_{1}(\cdot), u_{2}(\cdot)\right) \\
& =\mathbb{E} \int_{0}^{T}\left[l_{i}\left(t, \Theta^{\varepsilon}(t), u^{\varepsilon}(t)\right)-l_{i}(t, \bar{\Theta}(t), \bar{u}(t))\right] d t \\
& +\mathbb{E}\left[\gamma_{i}\left(y^{\varepsilon}(0)\right)-\gamma_{i}(\bar{y}(0))\right] \\
& +E\left[\phi_{i}\left(x^{\epsilon}(T)\right)-\phi_{i}(\bar{x}(T))\right] \\
& =\mathbb{E} \int_{0}^{T}\left[H_{i}\left(t, \Theta^{\varepsilon}(t), u^{\varepsilon}(t), \Lambda_{i}^{\bar{u}}(t)\right)\right. \\
& \left.-H_{i}\left(t, \bar{\Theta}(t), \bar{u}(t), \Lambda_{i}^{\bar{u}}(t)\right)\right] d t \\
& -\mathbb{E} \int_{0}^{T}\left\langle k_{i}^{\bar{u}}(t),-\left(f\left(t, \Theta^{\varepsilon}(t), u^{\varepsilon}(t)\right)\right.\right. \\
& -f(t, \bar{\Theta}(t), \bar{u}(t)))\rangle d t \\
& -\mathbb{E} \int_{0}^{T}\left\langle p_{i}^{\bar{u}}(t), b\left(t, \Theta^{\varepsilon}(t), u^{\varepsilon}(t)\right)\right. \\
& -b(t, \bar{\Theta}(t), \bar{u}(t))\rangle d t \\
& -\mathbb{E} \int_{0}^{T}\left\langle q_{i}^{\bar{u}}(t), \sigma\left(t, \Theta^{\varepsilon}(t), u^{\varepsilon}(t)\right)\right. \\
& -\sigma(t, \bar{\Theta}(t), \bar{u}(t))\rangle d t \\
& +\mathbb{E}\left[\gamma_{i}\left(y^{\varepsilon}(0)\right)-\gamma_{i}(\bar{y}(0))\right] \\
& +E\left[\phi_{i}\left(x^{\epsilon}(T)\right)-\phi_{i}(\bar{x}(T))\right] .
\end{aligned}
$$

Now putting (11) and (13) into (14), we deduce that (10) holds. The proof is complete. 


\section{A Variational Formula for Stochastic Differential Games}

In this section, we will obtain a directional derivative at a given admissible control process in some given control process direction. The choice of the given control process direction depends on the convexity of the control domain $U_{1} \times U_{2}$. If the control domain $U_{1} \times U_{2}$ is convex, a classical way of treating such a problem consists of using the convex perturbation method. More precisely, if $\left(\bar{u}_{1}(\cdot), \bar{u}_{2}(\cdot)\right)$ is a given admissible control and $\left(v_{1}(\cdot), v_{2}(\cdot)\right)$ is an arbitrary given admissible control, we can define a convex perturbed admissible control as

$$
u_{i}^{\varepsilon}(\cdot):=\bar{u}_{i}(\cdot)+\varepsilon\left(v_{i}(\cdot)-\bar{u}_{i}(\cdot)\right), \quad i=1,2,
$$

where $\varepsilon$ is a sufficiently small positive constant. Then one can prove the cost functional $J_{i}\left(u_{1}(\cdot), u_{2}(\cdot)\right)$ is Gâteaux differentiable at $\bar{u}_{i}(\cdot)$ in the direction $v_{i}(\cdot)-\bar{u}_{i}(\cdot),(i=1,2)$ and get a local stochastic maximum principle for openloop Nash equilibrium points; see, for example, [13, 14]. Different from $[13,14]$, our control domain in the present paper is not necessarily convex, so the convex perturbed control $u_{i}^{\varepsilon}(\cdot)$ may no longer be admissible and the convex perturbation method cannot be used to obtain the corresponding variational formula and maximum principle. A classical way of treating the nonconvex control domain consists of using the spike variations perturbation method. More precisely, let $(\bar{u}(\cdot) ; \bar{\Theta}(\cdot))=\left(\bar{u}_{1}(\cdot), \bar{u}_{2}(\cdot) ; \bar{x}(\cdot), \bar{y}(\cdot), \bar{z}(\cdot)\right)$ be any given admissible pair with the corresponding adjoint process $\Lambda_{i}^{\bar{u}}(\cdot):=\left(k_{i}^{\bar{u}}(\cdot), p_{i}^{\bar{u}}(\cdot), q_{i}^{\bar{u}}(\cdot)\right) \in \mathbb{M}^{2}[0, T], i=1,2$. We define the following spike variations:

$$
u_{i}^{\tau, \varepsilon}(t):= \begin{cases}u_{i}(t), & \text { if } t \in[\tau, \tau+\varepsilon] \\ \bar{u}_{i}(t), & \text { otherwise, }\end{cases}
$$

with fixed $\tau \in[0, T)$, sufficiently small positive $\varepsilon$, and any given admissible control $u_{i}(\cdot) \in \mathscr{A}_{1}, i=1,2$.

Now we state the following variational formula for the cost functional (2) associated with the spike variation (16) in a unified way.

Theorem 4. Under Assumption 1, one has a variational formula for the cost functional (5) and (6) as follows:

$$
\begin{aligned}
& \left.\frac{d}{d \varepsilon} J_{1}\left(u_{1}^{\tau, \varepsilon}(\cdot), \bar{u}_{2}(\cdot)\right)\right|_{\varepsilon=0} \\
& =\lim _{\varepsilon \rightarrow 0} \frac{J_{1}\left(u_{1}^{\tau, \varepsilon}(\cdot), \bar{u}_{2}(\cdot)\right)-J_{1}\left(\bar{u}_{1}(\cdot), \bar{u}_{2}(\cdot)\right)}{\varepsilon} \\
& =\mathbb{E}\left[H_{1}\left(\tau, \bar{\Theta}(\tau), u_{1}(\tau), \bar{u}_{2}(\tau), \Lambda_{1}^{\bar{u}}(\tau)\right)\right. \\
& \left.\quad-H_{1}\left(\tau, \bar{\Theta}(\tau), \bar{u}_{1}(\tau), \bar{u}_{2}(\tau), \Lambda_{1}^{\bar{u}}(t)\right)\right],
\end{aligned}
$$

$$
\begin{aligned}
\left.\frac{d}{d \varepsilon} J_{2}\left(\bar{u}_{1}(\cdot), u_{2}^{\tau, \varepsilon}(\cdot)\right)\right|_{\varepsilon=0} & \\
= & \lim _{\varepsilon \rightarrow 0} \frac{J_{2}\left(\bar{u}_{1}(\cdot), u_{2}^{\tau, \varepsilon}(\cdot)\right)-J_{2}\left(\bar{u}_{1}(\cdot), \bar{u}_{2}(\cdot)\right)}{\varepsilon} \\
= & \mathbb{E}\left[H_{2}\left(\tau, \bar{\Theta}(\tau), \bar{u}_{1}(\tau), u_{2}(\tau), \Lambda_{1}^{\bar{u}}(\tau)\right)\right. \\
& \left.-H_{2}\left(\tau, \bar{\Theta}(\tau), \bar{u}_{1}(\tau), \bar{u}_{2}(\tau), \Lambda_{1}^{\bar{u}}(\tau)\right)\right] .
\end{aligned}
$$

Proof. We first prove the equality (17). Let $\Theta^{1, \varepsilon}(\cdot)=$ $\left(x^{1, \varepsilon}(\cdot), y^{1, \varepsilon}(\cdot), z^{1, \varepsilon}(\cdot)\right)$ be the state process corresponding to the admissible control $\left(u_{1}^{\tau, \varepsilon}(\cdot), \bar{u}_{2}(\cdot)\right)$. Under Assumption 1 , by the continuous dependence theory of FBSDEs (see Proposition 3.2 in [16]), we have

$$
\left\|\Theta^{1, \varepsilon}(\cdot)-\Theta^{\bar{u}}(\cdot)\right\|_{\mathbb{M}^{2}} \leq C \varepsilon^{2} .
$$

In Theorem 3, replacing $\left(u^{\varepsilon}(\cdot) ; \Theta^{\varepsilon}(\cdot)\right)$ by $\left(u_{1}^{\tau, \varepsilon}(\cdot), \bar{u}_{2}(\cdot) ; \Theta^{1, \varepsilon}(\cdot)\right)$, we have

$$
\begin{gathered}
J_{1}\left(u_{1}^{\tau, \varepsilon}(\cdot), \bar{u}_{2}(\cdot)\right)-J_{1}\left(\bar{u}_{1}(\cdot), \bar{u}_{2}(\cdot)\right) \\
=\mathbb{E} \int_{0}^{T}\left[H_{i}\left(t, \bar{\Theta}(t), u_{1}^{\tau, \varepsilon}(t), \bar{u}_{2}(t), \Lambda_{i}^{\bar{u}}(t)\right)\right. \\
-H_{i}\left(t, \bar{\Theta}(t), \bar{u}_{1}(t), \bar{u}_{2}(t), \Lambda_{i}^{\bar{u}}(t)\right) \\
+H_{i}\left(t, \Theta^{\varepsilon}(t), u_{1}^{\tau, \varepsilon}(t), \bar{u}_{2}(t), \Lambda_{i}^{\bar{u}}(t)\right) \\
-H_{i}\left(t, \bar{\Theta}(t), u_{1}^{\tau, \varepsilon}(t), \bar{u}_{2}(t), \Lambda_{i}^{\bar{u}}(t)\right) \\
-\left\langle\bar{H}_{i x}(t), x^{\varepsilon}(t)-\bar{x}(t)\right\rangle \\
-\left\langle\bar{H}_{i y}(t), y^{\varepsilon}(t)-\bar{y}(t)\right\rangle \\
\left.-\left\langle\bar{H}_{i z}(t), z^{\varepsilon}(t)-\bar{z}(t)\right\rangle\right] d t \\
+\mathbb{E}\left[\gamma_{i}\left(y^{\varepsilon}(0)\right)-\gamma_{i}(\bar{y}(0))-\gamma_{i y}(\bar{y}(0))\right. \\
\left.\cdot\left(y^{\varepsilon}(0)-\bar{y}(0)\right)\right] \\
+\mathbb{E}\left[\phi_{i}\left(x^{\epsilon}(T)\right)-\phi_{i}(\bar{x}(T))-\phi_{i x}(\bar{x}(T))\right. \\
\left.\cdot\left(x^{\varepsilon}(T)-\bar{x}(T)\right)\right] \\
\left.-\quad\left\langle h_{x}(\bar{x}(T))\left(x^{\varepsilon}(T)-\bar{x}(T)\right), k_{i}^{\bar{u}}(T)\right\rangle\right] .
\end{gathered}
$$

Combining (19) and Assumption 1, by Taylor Expansions on $H$ and the dominated convergence theorem, from (20) we conclude that

$$
\begin{aligned}
J_{1}\left(u_{1}^{\tau, \varepsilon}(\cdot), \bar{u}_{2}(\cdot)\right)-J_{1}\left(\bar{u}_{1}(\cdot), \bar{u}_{2}(\cdot)\right) \\
=\mathbb{E} \int_{0}^{T}\left[H_{1}\left(t, \bar{\Theta}(t), u_{1}^{\tau, \varepsilon}(t), \bar{u}_{2}(t), \Lambda_{1}^{\bar{u}}(t)\right)\right. \\
\left.\quad-H_{1}\left(t, \bar{\Theta}(t), \bar{u}_{1}(t), \bar{u}_{2}(t), \Lambda_{1}^{\bar{u}}(t)\right)\right] d t+o(\varepsilon)
\end{aligned}
$$




$$
\begin{aligned}
=\varepsilon \mathbb{E}[ & H_{1}\left(\tau, \bar{\Theta}(\tau), u_{1}(\tau), \bar{u}_{2}(\tau), \Lambda_{1}^{\bar{u}}(\tau)\right) \\
& \left.-H_{1}\left(\tau, \bar{\Theta}(\tau), \bar{u}_{1}(\tau), \bar{u}_{2}(\tau), \Lambda_{1}^{\bar{u}}(t)\right)\right]+o(\varepsilon),
\end{aligned}
$$

which imply that (17) holds.

Similarly, we can prove that (18) holds. The proof is complete.

\section{Stochastic Maximum Principle}

In this section, applying the variational formulas (17) and (18), we will state and prove the global maximum principle for the Nash equilibrium points of Problem 2.

Theorem 5. Under Assumption 1, let $\bar{u}(\cdot)=\left(\bar{u}_{1}(\cdot), \bar{u}_{2}(\cdot)\right)$ be a Nash equilibrium point of Problem 2 with the state process $\bar{\Theta}(\cdot)=(\bar{x}(\cdot), \bar{y}(\cdot), \bar{z}(\cdot))$. Let $\Lambda_{i}(\cdot)=\left(p_{i}(\cdot), q_{i}(\cdot), k_{i}(\cdot)\right)(i=1,2)$ be the unique solution of the adjoint equation (8) corresponding to $(\bar{u}(\cdot) ; \bar{\Theta}(\cdot))$. Then

$$
\begin{aligned}
H_{1} & \left(\tau, \bar{\Theta}(\tau), \bar{u}_{1}(\tau), \bar{u}_{2}(\tau), \Lambda_{1}(\tau)\right) \\
= & \min _{u_{1} \in U_{1}} H_{1}\left(\tau, \bar{\Theta}(\tau), u_{1}, \bar{u}_{2}(\tau), \Lambda_{1}(\tau)\right), \\
H_{2} & \left(\tau, \bar{\Theta}(\tau), \bar{u}_{1}(\tau), \bar{u}_{2}(\tau), \Lambda_{2}(\tau)\right) \\
& =\min _{u_{2} \in U_{2}} H_{2}\left(\tau, \bar{\Theta}(\tau), \bar{u}_{1}(\tau), u_{2}, \Lambda_{2}(\tau)\right)
\end{aligned}
$$

hold for a.e. $(\tau, \omega) \in[0, T] \times \Omega$.

Proof. Since $\bar{u}(\cdot)=\left(\bar{u}_{1}(\cdot), \bar{u}_{2}(\cdot)\right)$ is a Nash equilibrium point of Problem 2, by (5), we have

$$
J_{1}\left(u_{1}^{\tau, \varepsilon}(\cdot), \bar{u}_{2}(\cdot)\right)-J_{1}\left(\bar{u}_{1}(\cdot), \bar{u}_{2}(\cdot)\right) \geq 0
$$

Using the notation in Theorem 3, for any arbitrary admissible control $\left(u_{1}(\cdot), u_{2}(\cdot)\right)$ and $t \in[0, T]$, we have

$$
\begin{aligned}
& \mathbb{E}\left[H_{1}\left(\tau, \bar{\Theta}(\tau), u_{1}(\tau), \bar{u}_{2}(\tau), \Lambda_{1}^{\bar{u}}(\tau)\right)\right. \\
& \left.-H_{1}\left(t, \bar{\Theta}(\tau), \bar{u}_{1}(\tau), \bar{u}_{2}(\tau), \Lambda_{1}^{\bar{u}}(\tau)\right)\right] \geq 0
\end{aligned}
$$

which implies that (22) holds.

Similarly, we can prove that (23) holds. The proof is complete.

\section{An Example: Linear Quadratic Case}

In this section, we work out an example of linear quadratic nonzero-sum differential games to illustrate our stochastic maximum principle. More precisely, consider the following one-dimensional linear fully coupled forward-backward stochastic system:

$$
\begin{aligned}
x(t)= & a+\int_{0}^{t}\left[A(s) x(s)-B(s) y(s)+D_{1}(s) u_{1}(s)\right. \\
& \left.+D_{2}(s) u_{2}(s)\right] d s \\
& +\int_{0}^{t}[G(s) x(s)] d B(s), \\
y(t)= & M x(T)+\int_{t}^{T}[A(s) y(s)+H(s) x(s)+G(s) z(s) \\
& -\int_{t}^{T} z(s) d B(s),
\end{aligned}
$$

with the quadratic cost functional

$$
\begin{aligned}
J_{i}\left(u_{1}(\cdot), u_{2}(\cdot)\right)=E[ & {\left[\int_{0}^{T} L_{i}(t) u_{i}^{2}(t) d t\right.} \\
& \left.+Q_{i} x^{2}(T)+N_{i} y^{2}(0)\right], \quad i=1,2,
\end{aligned}
$$

where $A, B, D_{1}, D_{2}, G, H, I_{1}, I_{2}, L_{1}$, and $L_{2}$ are onedimensional deterministic bounded measurable functions and $M, N_{1}, N_{2}, Q_{1}$, and $Q_{2}$ are constants. Also assume $B \geq 0$, $N_{1} \geq 0, N_{2} \geq 0, Q_{1} \geq 0, Q_{2} \geq 0, M \geq \beta, L_{1} \geq \beta, L_{2} \geq \beta$, $H>\beta$, where $\beta>0$ is a positive constant.

Under the above assumptions on the coefficients of (26) and (27), it is easy to check that, for any admissible $\left(u_{1}(\cdot), u_{2}(\cdot)\right) \in \mathscr{A}_{1} \times A_{2}$, the state system (26) has a unique solution and the corresponding stochastic differential game problem is well defined. For this case, the corresponding $H$ becomes

$$
\begin{aligned}
H_{i}( & \left.t, x, y, z, u_{1}, u_{2}, p_{i}, q_{i}, k_{i}\right) \\
= & -k_{i}\left[A(t) y+H(t) x+G(t) z+I_{1}(t) u_{1}+I_{2}(t) u_{2}\right] \\
& +p_{i}\left[A(t) x-B(t) y+D_{1}(t) u_{1}+D_{2}(t) u_{2}(s)\right] \\
& +q_{i} G(t) x+L_{i}(t) u_{i}^{2}(t), \quad i=1,2
\end{aligned}
$$

The corresponding adjoint equation associated with an admissible control pair $\left(\bar{u}_{1}(\cdot), \bar{u}_{2}(\cdot) ; \bar{x}(\cdot), \bar{y}(\cdot), \bar{z}(\cdot)\right)$ becomes

$$
\begin{aligned}
k_{i}(t)= & -2 N_{i} \bar{y}(0)+\int_{0}^{t}\left[A(s) k_{i}(s)+B(s) p_{i}(s)\right] d s \\
& +\int_{0}^{t} G(s) k_{i}(s) d B(s),
\end{aligned}
$$




$$
\begin{aligned}
p_{i}(t)= & -M k_{i}(T)+2 Q_{i} \bar{x}(T) \\
& +\int_{t}^{T}\left[A(s) p_{i}(s)+G(s) q_{i}(s)-H(s) k_{i}(s)\right] d s \\
& -\int_{t}^{T} q_{i}(s) d B(s), \quad i=1,2 .
\end{aligned}
$$

It is easy to check that the state system (26) has a unique solution $\left(p^{i}(\cdot), q^{i}(\cdot), k^{i}(\cdot)\right), i=1,2$.

Suppose that $\left(\bar{u}_{1}(\cdot), \bar{u}_{2}(\cdot)\right)$ is a Nash equilibrium point. By the maximum principle (see Theorem 5), putting the optimality conditions (22) and (23), the corresponding state equation (26), and the adjoint equations (28) associated with $\left(\bar{u}_{1}(\cdot), \bar{u}_{2}(\cdot)\right)$ together, we obtain the following optimality system for a Nash equilibrium point:

$$
\begin{aligned}
& x(t)=a+\int_{0}^{t}\left[A(s) x(s)-B(s) y(s)+D_{1}(s) u_{1}(s)\right. \\
& \left.+D_{2}(s) u_{2}(s)\right] d s \\
& +\int_{0}^{t} G(s) x(s) d B(s) \text {, } \\
& y(t)=M x(T)+\int_{t}^{T}[A(s) y(s)+H(s) x(s)+G(s) z(s) \\
& \left.+I_{1}(s) u_{1}(s)+I_{2}(s) u_{2}(s)\right] d s \\
& -\int_{t}^{T} z(s) d B(s) \\
& k_{i}(t)=-2 N_{i} \bar{y}(0)+\int_{0}^{t}\left[A(s) k_{i}(s)+B(s) p_{i}(s)\right] d s \\
& +\int_{0}^{t} G(s) k_{i}(s) d B(s) \\
& p_{i}(t)=-M k_{i}(T)+2 Q_{i} \bar{x}(T) \\
& +\int_{t}^{T}\left[A(s) p_{i}(s)+G(s) q_{i}(s)-H(s) k_{i}(s)\right] d s \\
& -\int_{t}^{T} q_{i}(s) d B(s) \\
& H_{1}\left(t, \bar{\Theta}(t), \bar{u}_{1}(t), \bar{u}_{2}(t), \bar{\Lambda}_{1}(t)\right) \\
& =\min _{u_{1} \in U_{1}} H_{1}\left(t, \bar{\Theta}(t), u_{1}, \bar{u}_{2}(t), \bar{\Lambda}_{1}(t)\right), \\
& H_{2}\left(t, \bar{\Theta}(t), \bar{u}_{1}(t), \bar{u}_{2}(t), \bar{\Lambda}_{2}(t)\right) \\
& =\min _{u_{2} \in U_{2}} H_{2}\left(t, \bar{\Theta}(t), \bar{u}_{1}(t), u_{2}, \bar{\Lambda}_{2}(t)\right), \\
& t \in[0, T], \quad i=1,2 .
\end{aligned}
$$

This is called coupled forward-backward stochastic differential equations with double dimensions (DFBSDE for short).
Note that the coupling comes from the two last relations (which is the maximum condition). We also refer to $\mathrm{Yu}$ [17] for the general theory of this kind equation. The 8-tuple $\left(u_{1}(\cdot)\right.$, $\left.u_{2}(\cdot), x(\cdot), y(\cdot), z(\cdot), p(\cdot), q(\cdot), k(\cdot)\right)$ of $\mathscr{F}$-adapted processes satisfying the above is called an adapted solution of (30). We now look at the sufficiency of the existence of a Nash equilibrium point.

Theorem 6. Suppose that $\left(\bar{u}_{1}(\cdot), \bar{u}_{2}(\cdot), \bar{x}(\cdot), \bar{y}(\cdot), \bar{z}(\cdot), p(\cdot)\right.$, $q(\cdot), k(\cdot))$ is an adapted solution to DFBSDE (30). Then $\left(\bar{u}_{1}(\cdot), \bar{u}_{2}(\cdot)\right)$ is a Nash equilibrium point.

Proof. Let $\left(\bar{u}_{1}(\cdot), \bar{u}_{2}(\cdot), \bar{x}(\cdot), \bar{y}(\cdot), \bar{z}(\cdot), p(\cdot), q(\cdot), k(\cdot)\right)$ be an adapted solution to DFBSDE (30). For any admissible control pair $\left(u_{1}(\cdot), u_{2}(\cdot)\right) \in \mathscr{A}_{1} \times A_{2}$, from Theorem 3 , we have

$$
\begin{aligned}
& J_{i}\left(u_{1}(\cdot), \bar{u}_{2}(\cdot)\right)-J_{i}\left(\bar{u}_{1}(\cdot), \bar{u}_{2}(\cdot)\right) \\
& =\mathbb{E} \int_{0}^{T}\left[H_{1}\left(t, \bar{\Theta}(t), u_{1}(t), \bar{u}_{2}(t), \Lambda_{i}^{\bar{u}}(t)\right)\right. \\
& \left.-H_{i}\left(t, \bar{\Theta}(t), \bar{u}_{1}(t), \bar{u}_{2}(t), \Lambda_{i}^{\bar{u}}(t)\right)\right] d t \\
& +\mathbb{E}\left[N_{1}\left(y^{\left(u_{1}, \bar{u}_{2}\right)}(0)\right)^{2}-N_{1}(\bar{y}(0))^{2}\right. \\
& \left.-2 N_{1} \bar{y}(0)\left(y^{\left(u_{1}, \bar{u}_{2}\right)}(0)-\bar{y}(0)\right)\right] \\
& +\mathbb{E}\left[Q_{1}\left(x^{\left(u_{1}, \bar{u}_{2}\right)}(T)\right)^{2}-Q_{1} x^{2}(T)\right. \\
& \left.-2 Q_{1} x^{2}(T) \cdot\left(x^{\left(u_{1}, \bar{u}_{2}\right)}(T)-\bar{x}(T)\right)\right] \\
& =\mathbb{E} \int_{0}^{T}\left[H_{1}\left(t, \bar{\Theta}(t), u^{\varepsilon}(t), \Lambda_{i}^{\bar{u}}(t)\right)\right. \\
& \left.-H_{1}\left(t, \bar{\Theta}(t), \bar{u}(t), \Lambda_{1}^{\bar{u}}(t)\right)\right] d t \\
& +\mathbb{E}\left[N_{1}\left(y^{\left(u_{1}, \bar{u}_{2}\right)}(0)-\bar{y}(0)\right)^{2}\right] \\
& +\mathbb{E}\left[Q_{1}\left(x^{\left(u_{1}, \bar{u}_{2}\right)}(T)-x(T)\right)^{2}\right] \\
& \geq 0 \text {, }
\end{aligned}
$$

which implies that

$$
J_{1}\left(\bar{u}_{1}(\cdot), \bar{u}_{2}(\cdot)\right)=\inf _{u_{1}(\cdot) \in \mathscr{A}_{1}} J_{1}\left(u_{1}(\cdot), \bar{u}_{2}(\cdot)\right) .
$$

Similarly, we can get

$$
J_{2}\left(\bar{u}_{1}(\cdot), \bar{u}_{2}(\cdot)\right)=\inf _{u_{2}(\cdot) \in \mathscr{A}_{2}} J_{2}\left(\bar{u}_{1}(\cdot), u_{2}(\cdot)\right) .
$$

Therefore, $\left(\bar{u}_{1}(\cdot), \bar{u}_{2}(\cdot)\right)$ is a Nash equilibrium point.

Proposition 7. Suppose that $U_{1} \times U_{2}=\mathbb{R} \times \mathbb{R}$ and $\left(\bar{u}_{1}(\cdot), \bar{u}_{2}(\cdot), \bar{x}(\cdot), \bar{y}(\cdot), \bar{z}(\cdot), p(\cdot), q(\cdot), k(\cdot)\right)$ is an adapted solution to DFBSDE (30). Then $\left(\bar{u}_{1}(\cdot), \bar{u}_{2}(\cdot)\right)$ has the following representation:

$$
\bar{u}_{i}(t)=\frac{1}{2 L_{i}(t)}\left[k(t) I_{i}(t)-p_{i}(t) D_{i}(t)\right], \quad i=1,2 .
$$


Proof. From Theorem 6, we deduce that $\left(\bar{u}_{1}(\cdot), \bar{u}_{2}(\cdot)\right)$ is a Nash equilibrium point. Then from $U_{1} \times U_{2}=\mathbb{R} \times \mathbb{R}$ and the optimality conditions (22) and (23), it follows that

$$
\begin{aligned}
& H_{1 u_{1}}\left(t, \bar{\Theta}(t), \bar{u}_{1}(t), \bar{u}_{2}(t), \bar{\Lambda}_{1}(t)\right)=0, \\
& H_{2 u_{2}}\left(t, \bar{\Theta}(t), \bar{u}_{1}(t), \bar{u}_{2}(t), \bar{\Lambda}_{2}(t)\right)=0,
\end{aligned}
$$

which imply that

$$
\bar{u}_{i}(t)=\frac{1}{2 L_{i}(t)}\left[k(t) I_{i}(t)-p_{i}(t) D_{i}(t)\right], \quad i=1,2 .
$$

The proof is complete.

Remark 8. In summary, DFBSDE (30) completely characterizes the Nash equilibrium point. Therefore, solving the differential game problem is equivalent to solving the DFBSDE (30). Moreover, a candidate equilibrium point can be given by (34). We refer the reader to Yu [17] for the theory of solvability to DFBSDE (30). Since the linear quadratic control problem is an important and fascinating class of stochastic control ones, and the theoretical results of this problem have lots of significant impacts on a wide range of engineering, managing, and financial applications, in the future, we will focus on the study of financial application as the reviewers suggest.

\section{Conclusion}

In this paper, a two-person nonzero-sum differential game is studied for a fully coupled forward-backward stochastic system with the control process $u(\cdot)$ not appearing in the forward diffusion term, but the control domain not necessarily convex. For this case, we obtain a variation formula for the cost functional. As an application, the maximum principle for open-loop Nash equilibrium points is established. Finally, we work out an example of linear quadratic nonzero-sum differential games to illustrate our stochastic maximum principle. As the reviewers suggest, our system discussed in this paper may be extended to the discontinuous system such Markov-switching jump-diffusion system for which the optimal control problems and differential games have extensive applications in industry and finance (see, e.g., $[18,19]$ and the references therein). Some investigations on this topic will be studied and carried out in our future publications.

\section{Conflict of Interests}

The author declares that there is no conflict of interests regarding the publication of this paper.

\section{Acknowledgment}

This work was partially supported by the National Natural Science Foundation of China (11101140, 11301177), the China Postdoctoral Science Foundation (2011M500721, 2012T50391), and the Natural Science Foundation of Zhejiang Province (nos. Y6110775 and Y6110789).

\section{References}

[1] J.-M. Bismut, "Conjugate convex functions in optimal stochastic control," Journal of Mathematical Analysis and Applications, vol. 44, no. 3, pp. 384-404, 1973.

[2] É. Pardoux and S. G. Peng, "Adapted solution of a backward stochastic differential equation," Systems \& Control Letters, vol. 14, no. 1, pp. 55-61, 1990.

[3] S. Tang, "General linear quadratic optimal stochastic control problems with random coefficients: linear stochastic Hamilton systems and backward stochastic Riccati equations," SIAM Journal on Control and Optimization, vol. 42, no. 1, pp. 53-75, 2003.

[4] B. Øksendal and A. Sulem, "Maximum principles for optimal control of forward-backward stochastic differential equations with jumps," SIAM Journal on Control and Optimization, vol. 48, no. 5, pp. 2945-2976, 2009/10.

[5] B. Øksendal and A. Sulem, "Forward backward stochastic differential games and stochastic control under model uncertainty," Journal of Optimization Theory and Applications, 2012.

[6] W. S. Xu, "Stochastic maximum principle for optimal control problem of forward and backward system," Australian Mathematical Society Journal B, vol. 37, no. 2, pp. 172-185, 1995.

[7] Z. Wu, "Maximum principle for optimal control problem of fully coupled forward-backward stochastic systems," Systems Science and Mathematical Sciences, vol. 11, no. 3, pp. 249-259, 1998.

[8] J.-T. Shi and Z. Wu, "The maximum principle for fully coupled forward-backward stochastic control system," Acta Automatica Sinica, vol. 32, no. 2, pp. 161-169, 2006.

[9] Q. Meng, "A maximum principle for optimal control problem of fully coupled forward-backward stochastic systems with partial information," Science in China A, vol. 52, no. 7, pp. 1579-1588, 2009.

[10] J. Shi and Z. Wu, "Maximum principle for forward-backward stochastic control system with random jumps and applications to finance," Journal of Systems Science \& Complexity, vol. 23, no. 2, pp. 219-231, 2010.

[11] Z. Wu, "A general maximum principle for optimal control of forward-backward stochastic systems," Automatica, vol. 49, no. 5, pp. 1473-1480, 2013.

[12] J. Yong, "Optimality variational principle for controlled forward-backward stochastic differential equations with mixed initial-terminal conditions," SIAM Journal on Control and Optimization, vol. 48, no. 6, pp. 4119-4156, 2010.

[13] E. C. M. Hui and H. Xiao, "Maximum principle for differential games of forward-backward stochastic systems with applications," Journal of Mathematical Analysis and Applications, vol. 386, no. 1, pp. 412-427, 2012.

[14] M. Tang, "Maximum principle for non-zero sum stochasticdifferential games of fully coupled forward-backward stochastic systems," preprint.

[15] S. Peng and Z. Wu, "Fully coupled forward-backward stochastic differential equations and applications to optimal control," SIAM Journal on Control and Optimization, vol. 37, no. 3, pp. 825-843, 1999.

[16] Q. Lin, "Optimal control of coupled forward-backward stochastic system with jumps and related hamilton-jacobi-bellman equations," http://arxiv.org/abs/1111.4642.

[17] Z. Yu, "Linear-quadratic optimal control and nonzero-sum differential game of forward-backward stochastic system," Asian Journal of Control, vol. 14, no. 1, pp. 173-185, 2012. 
[18] B. Z. Temoçin and G.-W. Weber, "Optimal control of stochastic hybrid system with jumps: a numerical approximation," Journal of Computational and Applied Mathematics, vol. 259, pp. 443451, 2014.

[19] N. Azevedo, D. Pinheiro, and G. W. Weber, "Dynamic programming for a Markov-switching jumpCdiffusion," Journal of Computational and Applied Mathematics, vol. 267, pp. 1-19, 2014. 


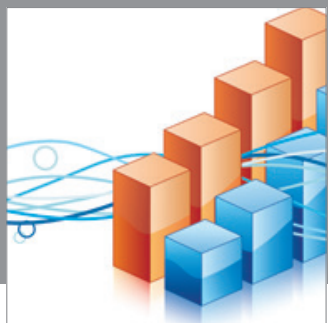

Advances in

Operations Research

mansans

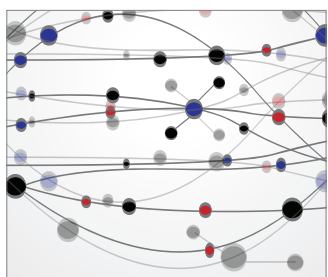

The Scientific World Journal
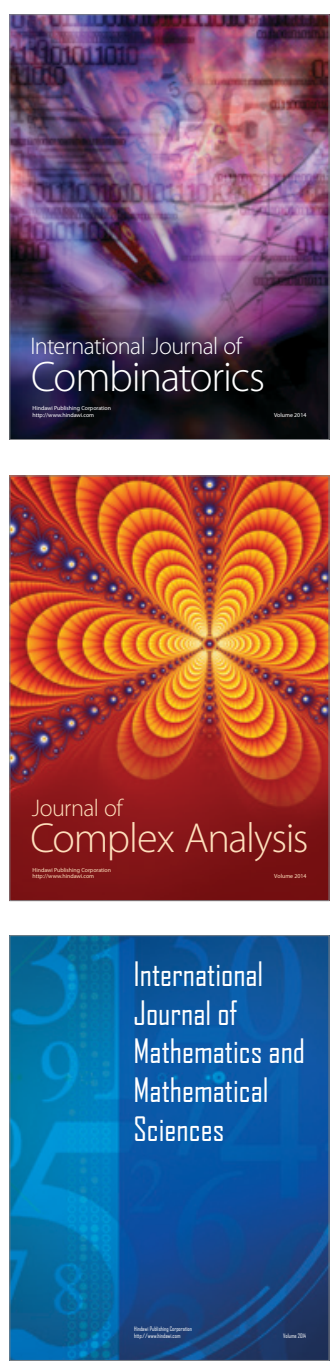
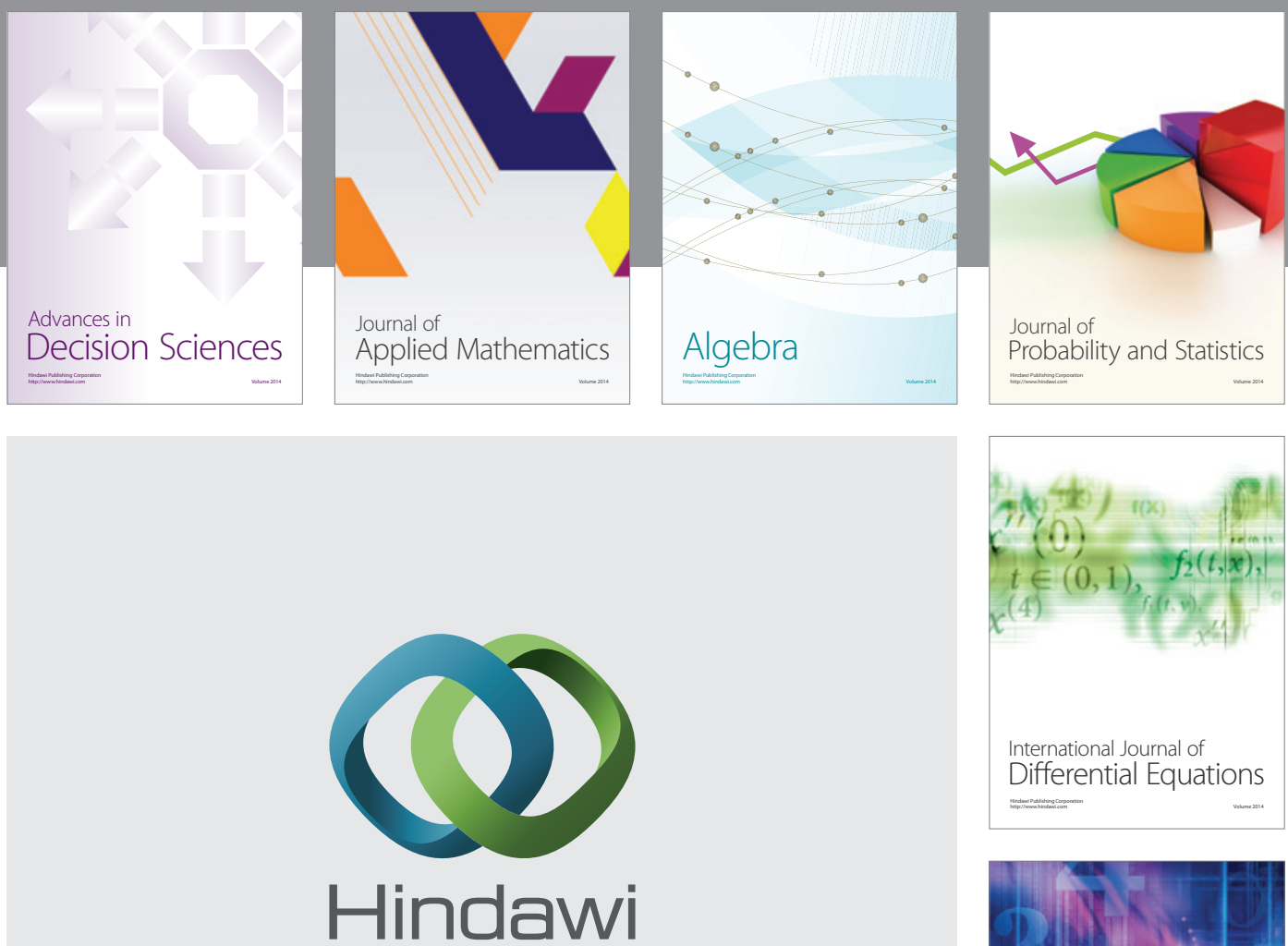

Submit your manuscripts at http://www.hindawi.com
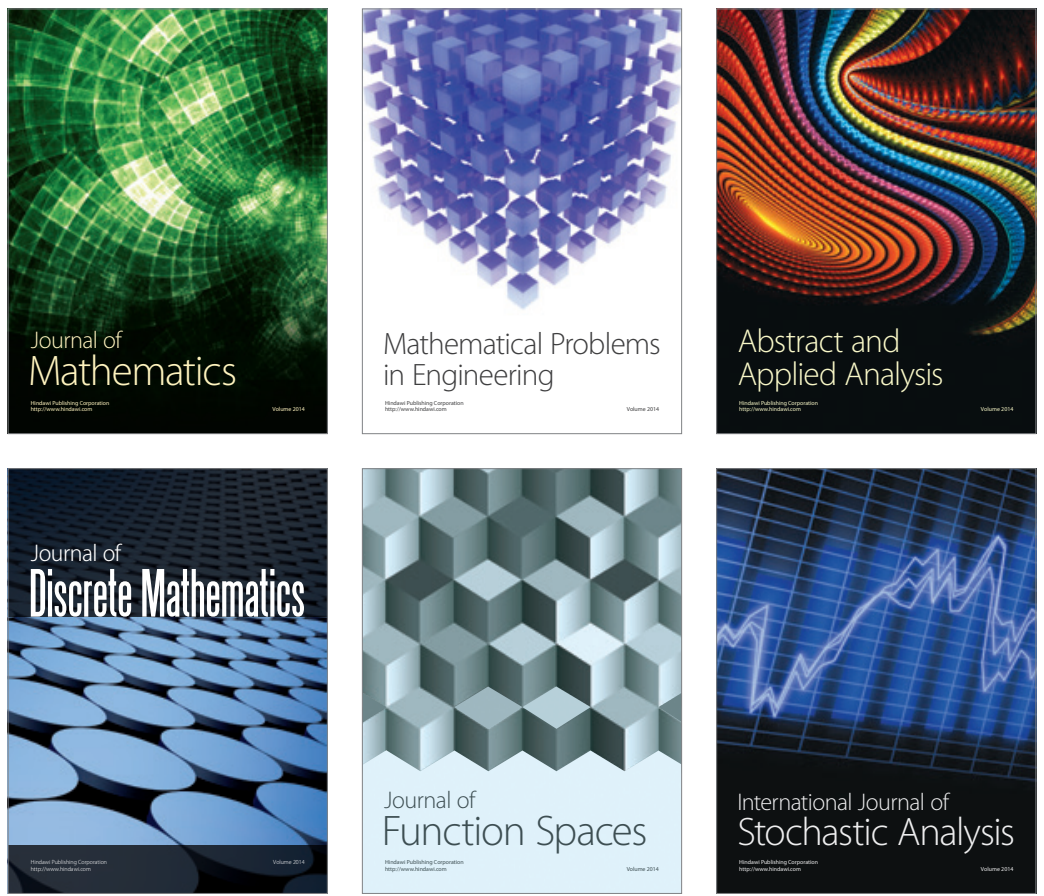

Journal of

Function Spaces

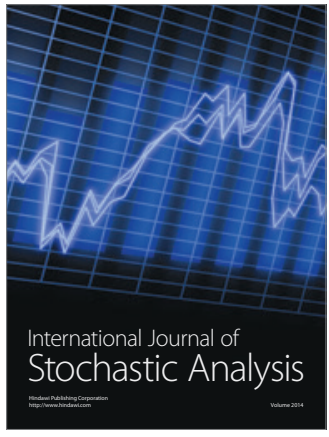

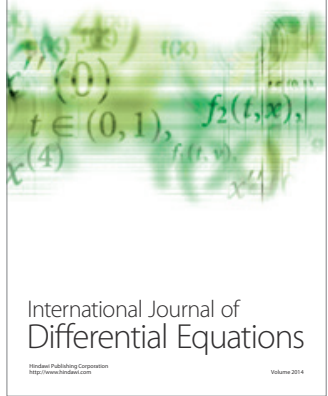
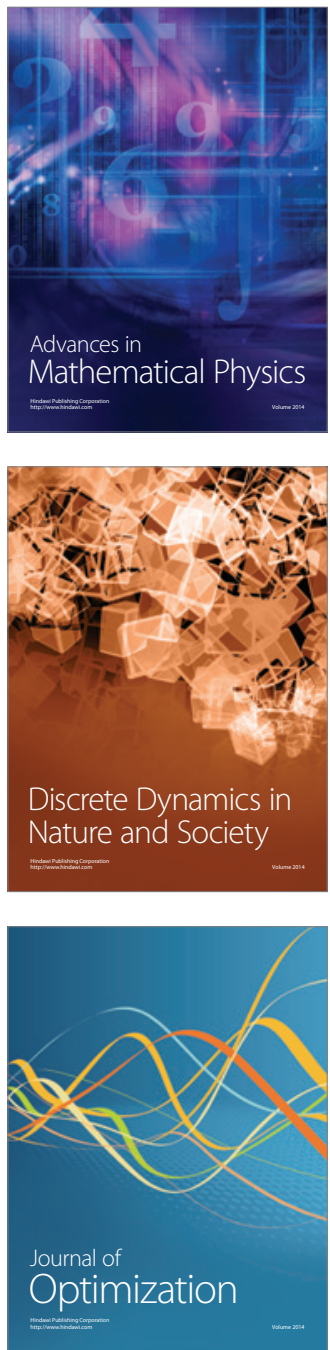